\title{
Contribuição da Fisioterapia Segundo Médicos e Enfermeiros das Unidades Básicas de Saúde da Família
}

\author{
Thais Fernanda Tortorelli Zarili ${ }^{1}$, Maria Dionísia do Amaral Dias $^{2}$
}

\begin{abstract}
RESUMO
Objetivo: Identificar a percepção de médicos e enfermeiros de Unidades Básicas de Saúde com Estratégia de Saúde da Família (UBSF) de Botucatu (SP), com relação aos principais obstáculos no processo de trabalho das equipes de Atenção Primária à Saúde (APS) e a contribuição das ações e serviços de Fisioterapia para o cuidado integral dos usuários. Materiais e Métodos: Estudo descritivo, transversal e abordagem qualitativa. Foi aplicado um questionário semiestruturado, formulado pelos pesquisadores, com 27 questões que abordam a caracterização do entrevistado, as dificuldades do processo de trabalho e a visão dos profissionais quanto à contribuição da fisioterapia no cuidado aos usuários. As respostas dicotômicas foram analisadas segundo a frequência e as respostas abertas segundo análise temática. Participaram da pesquisa 28 médicos e enfermeiros de todas as UBSFs do município. Resultados: Os temas que emergiram demonstram a sobrecarga de trabalho pelo excesso de demanda e dificuldades com relação ao próprio serviço, à equipe e à gestão municipal, que tem conhecimento quanto à atuação da Fisioterapia e sabe que há diversas demandas que necessitam dessa especialidade, assim como compreende a contribuição para a resolutividade dos casos atendidos no serviço e a melhoria da qualidade de vida do paciente. Conclusão: A inserção do fisioterapeuta na APS e a rede de serviços de Fisioterapia corroboram com a clínica ampliada enquanto ferramenta para o cuidado. O fortalecimento das suas ações deve estar em consonância com as necessidades do território e articulado com a equipe do serviço.
\end{abstract}

Palavras-chave: Fisioterapia. Saúde da família. Pesquisa qualitativa. Atenção primária à saúde.

CONTRIBUTION OF PHYSIOTHERAPY ACCORDING TO DOCTORS AND NURSES AT BASIC FAMILY HEALTH UNITS

\section{ABSTRACT}

Objective: Identify the perception of physicians and nurses in the Family Health of Botucatu (SP) about the main obstacles in the work process of Primary Health Care (PHC) teams and the contribution of Physical Therapy actions and services to the comprehensive care of users. Material and Methods: Study descriptive, cross-sectional design and qualitative approach. A semi-structured questionnaire was applied, formulated by the researcher, with 27 questions that address characterization of the interviewee, difficulties in the work process, the view of professionals regarding the contribution of Physiotherapy to users' health. The dichotomous responses were analyzed according to the frequency and the answers opened according to the thematic analysis. A total of 28 professionals, mostly female, participated in the study. Results: The themes that emerged demonstrate the overload of work due to the excess of demand and difficulties in relation to the service itself, from the team and from the municipal management, that there is knowledge about the performance of Physiotherapy and that there are several demands that require this specialty, as well as understanding the contribution to the resolution of the cases attended in the service and the improvement of the quality of life of the patient. Conclusion: The insertion of the physiotherapist in the PHC and Physical Therapy networks services corroborates with the extended clinic as a tool for the care. The strengthening of its actions must be in harmony with the needs of the territory and articulated with the staff of the service.

Keywords: Physical therapy. Family health. Qualitative research. Primary health care.

RECEBIDO EM: 5/11/2019

MODIFICAÇÕES SOLICITADAS EM: 22/2/2020

ACEITO EM: 23/4/2020

\footnotetext{
Doutorado em Saúde Coletiva pela Universidade Estadual Paulista Júlio de Mesquita Filho. Faculdade de Medicina de Botucatu/Unesp. http://lattes.cnpq. br/9931226404940771. https://orcid.org/0000-0002-0690-2334. thaisftzarili@gmail.com

2 Doutorado em Psicologia (Psicologia Social) pela Pontifícia Universidade Católica de São Paulo. Faculdade de Medicina de Botucatu/Unesp. http://lattes. cnpq.br/5242671242882106. https://orcid.org/0000-0002-0081-6769. dionisia@fmb.unesp.br
} 


\section{INTRODUÇÃO}

O apoio matricial pauta-se em uma lógica de trabalho horizontal entre os profissionais de saúde do Sistema Único de Saúde (SUS) de diferentes níveis de atenção da rede de atenção à saúde, com estratégia fortalecida pela atuação das equipes do Núcleo Ampliado de Saúde da Família (Nasf), modificando o modelo hierarquizado composto por mecanismos de referência e contrarreferência (BARROS et al., 2015; MENDES, 2011). Assim, a rede de atenção à saúde deve dialogar com a Atenção Primária à Saúde (APS) como retaguarda assistencial e pedagógica.

Diante do atual panorama da APS, com a republicação da Política Nacional de Atenção Básica em 2017, o Núcleo de Apoio à Saúde da Família foi rebatizado como Núcleo Ampliado de Saúde da Família e Atenção Básica, e passou seu escopo de atuação para além das Estratégia de Saúde da Família (ESF), incluindo, também, outras equipes de APS (MOROSINI; FONSECA; LIMA, 2018; BRASIL, 2017). O programa Previne Brasil (BRASIL, 2019), lançado no final de 2019, contudo, trouxe mudanças significativas no modelo de financiamento da Atenção Primária no SUS, que impactam, ainda, sobre o Nasf, pois não há o estímulo específico para o núcleo, o que vinha ocorrendo de forma crescente desde a sua criação, e fica a cargo do gestor local definir se utilizará seus recursos financeiros da Saúde para a construção dessas equipes e sua manutenção.

Diante deste cenário, o debate sobre a importância da inserção de outras categoriais multiprofissionais e da lógica de uma rede de atenção viva e em movimento, faz-se mais atual que nunca.

A ESF, idealizada em 1994 pelo Ministério da Saúde, constitui-se na Política Nacional de Atenção Básica (PNAB) como prioridade para sua expansão e consolidação, e possui como objetivo a reorganização da prática assistencial em substituição ao modelo tradicional de assistência (OMS, 2008; RODRIGUES; RAMIRES, 2015; BRASIL, 2017). Para tal, além da aproximação com as necessidades da população e o contexto familiar, almeja-se ampliar a resolutividade da demanda atendida e o impacto sobre a situação de saúde da população da área de abrangência (BRASIL, 2017).

A Integralidade é condicionada a um trabalho multiprofissional, interdisciplinar e em equipe, sob a ótica da gestão do cuidado integral do usuário e da coordenação pela APS do percurso do usuário pela rede de atenção (AITH et al., 2014). A APS realiza ações de promoção, proteção e recuperação da saúde e prevenção de agravos, assim como o atendimento da demanda espontânea, da realização das ações programáticas, coletivas e de vigilância à saúde (BRASIL, 2017), tendo como expectativa a resolutividade de $85 \%$ das demandas em saúde da população (MENDES, 2011; CUBAS et al., 2017; VASCONCELOS et al., 2018). Heidemann, Wosny e Boehs (2014), no entanto, destacam que o trabalho preventivo e de promoção da saúde no cotidiano das unidades de saúde é um desafio e, em muitas delas, permanece a lógica curativa e imediatista, a despeito dos princípios da ESF e recomendações explicitadas nas normas do SUS.

O envelhecimento populacional, o número de pessoas com deficiências e doenças crônicas, além das características do trabalho na sociedade capitalista, responsáveis pelo aumento das doenças osteomioarticulares, promoveram alterações substanciais no perfil demográfico e epidemiológico, constituindo-se como um desafio em saúde pública pelo impacto sobre a qualidade de vida de indivíduos e da coletividade. Este cenário empreende maiores esforços de gestores e profissionais de saúde em prol de atender às necessidades da população e à satisfação do usuário pelo atendimento recebido (CALICCHIO et al., 2018). Estas, dentre outras características, apontam para a necessidade da atuação multiprofissional no sentido de ampliar o sucesso das intervenções em situações complexas do processo saúde-doença.

Com o objetivo de aumentar a resolutividade da ESF, houve a criação, pelo Ministério da Saúde, dos Núcleos de Apoio à Saúde da Família (Nasf) em 2008, incluindo profissionais de diversas categorias, para atuarem em conjunto com a equipe mínima por meio de um processo de trabalho que reforce os pressupostos do SUS. Pretendeu-se, com a instituição do Nasf, contribuir para que a atenção em saúde vá além da lógica fragmentada, de modo a fortalecer a construção das redes de atenção e cuidado, assim como fazer com que os profissionais inseridos sejam agentes de transformação social (BRASIL, 2010; MELO et al., 2018).

O processo de trabalho do Nasf consiste em vincular suas ações totalmente à equipe de APS, atuando na própria unidade. Durante o trabalho, a equipe do Nasf pode realizar discussões de caso, consultas compartilhadas com outros membros da equipe, construção de projetos terapêuticos, participar na execução de educação permanente, entre outras atribuições (BRASIL, 2017; MELO et al., 2018).

A possibilidade de atuação multiprofissional no SUS, com especial destaque à inserção do fisioterapeuta, além de favorecer o cuidado integral do usuá- 
rio, também abriu novas perspectivas ao profissional desta área num campo de atuação diferente daquele no qual a fisioterapia é tradicionalmente conhecida e reconhecida: o da reabilitação (FERNANDES DE AMORIM et al., 2017). Somente em 2009 o Conselho Federal de Fisioterapia e Terapia Ocupacional (Coffito) reconheceu o campo da Saúde Coletiva como especialidade da fisioterapia (COFFITO, 2009), portanto passaram-se 40 anos desde a regulamentação da profissão para que o fisioterapeuta tivesse sua atuação e formação reconhecida neste campo.

Segundo Santos et al. (2014), o fisioterapeuta atua na APS na proteção, promoção e reabilitação do usuário e na adaptação do indivíduo na sociedade, visando à melhoria da qualidade de vida, realizando diagnóstico cineticofuncional em atendimento fisioterapêutico individual ou coletivo ou na assistência domiciliar. Também pode desenvolver ações continuadas, realizar encaminhamentos aos serviços de referência, incentivar e assessorar o controle social em saúde e ministrar palestras ou cursos.

Para o fisioterapeuta há o desafio da associação do seu saber profissional ao saber de outras áreas e, fundamentalmente, ao conhecimento popular, rompendo com o modelo biomédico, o qual incorpora a busca por atendimento quando em estado crônico e dependente de um tratamento ainda mais prolongado (ROCHA JÚNIOR; MOREIRA, 2016). A fisioterapia ainda permanece, em muitas ocasiões, atrelada ao atendimento individualizado, tanto no processo de avaliação quanto no de tratamento, focando sua atenção no modelo saúde-doença, estando, ainda, inserida na APS de forma modesta (OLIVEIRA et al., 2011; RIBEIRO; SOARES, 2014).

Para além da APS, o conjunto de serviços de fisioterapia municipais dispõe de instituições públicas ou não para atendimento fisioterapêutico aos usuários do SUS, sendo, assim, serviços de referência para encaminhamento. Há a necessidade de interação entre os profissionais da UBSF, do Nasf e dos serviços secundários e terciários que constituem as Redes de Atenção à Saúde (RAS), para atender às demandas e construir e manter a lógica de rede na atenção ao usuário.

Sendo crescente e recente a atuação da Fisioterapia na APS (RIBEIRO; SOARES, 2014), torna-se relevante compreender a sua inserção, sobretudo, quanto à organização dos serviços de fisioterapia disponíveis no sistema municipal de saúde e o perfil da demanda nas UBSFs, buscando auxiliar na fomentação de políticas públicas e programas. Assim, esse estudo objetiva identificar obstáculos no processo de trabalho de equipes de APS e as contribuições da fisioterapia para o cuidado integral dos usuários segundo a percepção de médicos e enfermeiros da Estratégia de Saúde da Família de Botucatu (SP).

\section{MATERIAIS E MÉTODOS}

A metodologia caracteriza-se como um estudo descritivo e exploratório, com delineamento transversal e abordagem qualitativa. A pesquisa foi aprovada pelo Comitê de Ética em Pesquisa da Faculdade de Medicina de Botucatu da Universidade Estadual Paulista (Processo no 4270/2012) e atende à Resolução 466/12 do Conselho Nacional de Saúde do Ministério da Saúde (Brasil) no tocante à autonomia, beneficência, não maleficência, justiça e equidade.

Optou-se pela abordagem qualitativa, que traz questões como valores e opiniões, ou seja, dados subjetivos, por possuir a característica adequada para aprofundar fenômenos complexos, fatos, processos individuais e de grupos, trazidos com maior abrangência de componentes.

O local do estudo foi o município de Botucatu (SP), com uma população de 127.370 mil habitantes, segundo dados do último censo (IBGE, 2010), sendo $48,5 \%$ do sexo masculino e $51,5 \%$ do feminino, constando como pertencentes à região urbana $122.718 \mathrm{e}$ rural 4.652. O Índice de Desenvolvimento Humano de Botucatu é de 0,822 , colocando-o no 202 - lugar no ranking dos municípios brasileiros. Possuía, à época da pesquisa, 11 UBSFs, sob gestão de uma organização social, e 9 espaços com atendimento fisioterapêutico na rede pública de saúde, contando com 19 fisioterapeutas. O Nasf (Tipo 1) iniciou suas atividades em janeiro de 2012 e, no momento da pesquisa, a equipe era composta por dois fisioterapeutas, que frequentavam quinzenalmente as unidades, e a organização do processo de trabalho configurava-se de diferentes formas em cada unidade. Para além das unidades sob modelo de ESF, o Nasf do município prestava apoio também às unidades básicas de saúde tradicionais, portanto cada fisioterapeuta tinha sob sua responsabilidade nove serviços de APS.

Os participantes foram selecionados de forma intencional. Foram convidados todos os médicos e enfermeiros que atuavam nas UBSFs. Os critérios de inclusão foram médicos e enfermeiros das UBSFs do município, por considerar-se serem esses os profissionais com responsabilidades diretas na atenção aos usuários e envolvidos em diagnósticos e encaminhamentos de pacientes. A rede de saúde no município contava com 15 médicos e 14 enfermeiros em 11 UBS- 
Fs. Foram contatados todos os profissionais e houve a recusa apenas de um médico em participar da pesquisa. Foram explicados os objetivos, riscos e benefícios da pesquisa, contidos no Termo de Consentimento Livre e Esclarecido (TCLE), o qual foi assinado por todos os participantes.

Para a coleta dos dados foi utilizado um questionário semiestruturado, autoaplicável e anônimo, formulado pelos pesquisadores, com 27 questões, assim classificadas: caracterização do entrevistado profissão, idade, sexo, formação complementar, tempo de trabalho na atual unidade e experiências anteriores; dificuldades do processo de trabalho; a visão dos profissionais quanto à contribuição da fisioterapia na saúde nos usuários; consolidação da equipe do Nasf no município e dos serviços de retaguarda de fisioterapia.

Após a entrega do questionário, o participante teve um prazo de 15 dias para devolução em envelope lacrado, e as respostas foram digitalizadas em Microsoft Excel. As questões fechadas do questionário foram analisadas conforme a frequência e as questões abertas com base em unidades temáticas, realizando-se a confrontação e diálogo entre os dados.

Com relação às questões de respostas abertas, foram analisadas conforme as seguintes etapas: codificação e identificação de temas; refinamento de temas e categorias; desenvolvimento de teoria e incorporação de conhecimento preexistente; e produção de relatórios. Os temas podem ser entendidos como "unidade de significação que se liberta naturalmente de um texto analisado segundo critérios relativos à teoria que serve de guia à leitura" (BARDIN, 1979).

Para a questão sobre as dificuldades no processo de trabalho emergiram 10 temas como resultado da análise: "Sobrecarga de Trabalho", "Serviços de Retaguarda", "Características do Território", "Gestão Municipal", "Equipe", "NASF", "Condições Materiais de Trabalho", "Descaracterização da Estratégia de Saúde da Família", "Usuários" e "Pesquisas".

Para a questão sobre a atenção em Fisioterapia no município, houve a definição das categorias "Recuperar a Saúde", "Promover Saúde", "Melhorar a Qualidade de Vida", "Colaborar com a Equipe" e "Melhorar a Qualidade da Assistência".

Quanto às expectativas relacionadas ao fisioterapeuta do Nasf, emergiram as categorias "Atividades Coletivas", "Qualidade da Assistência", "Colaboração com a Equipe", "Reabilitação", "Promoção/Prevenção", "Qualidade de Vida", "Articulação com outros Serviços" e "Carga Horária na Unidade".
E a última temática abordada em questão aberta foi sobre a expectativa ao encaminhar o usuário para serviço de retaguarda em fisioterapia. Houve a observação sobre as categorias "Reabilitação", "Resolutividade", "Contrarreferência", "Melhora da Qualidade de Vida do Paciente", "Promoção/Prevenção" e "Colaboração com a Equipe".

\section{RESULTADOS}

Participaram da pesquisa 28 profissionais (14 médicos e 14 enfermeiros), equivalente a 96,5\% dos que atuavam na ESF. Com relação à caracterização dos participantes deste estudo, $68,9 \%$ são do sexo feminino e $27,6 \%$ do masculino, com média de idade de 33,6 anos, com variação entre 24 e 50 anos.

Quanto à formação complementar realizada após o curso de Graduação, todos os respondentes realizaram alguma formação complementar, posto que 12 (42,8\%) respondentes possuem duas ou mais, o que pode significar grande contribuição para a execução do trabalho. Ressalta-se o fato de que em Botucatu a Faculdade de Medicina oferece cursos diversos em nível de Pós-Graduação, inclusive com caráter multiprofissional, o que pode facilitar aos profissionais o acesso a essa experiência de formação contínua.

A maioria dos participantes apresenta experiência anterior em serviços da APS: $85,7 \%$ em UBSFs e $39,3 \%$ em unidade básica tradicional. O tempo de permanência, em média, em outra UBSF foi de dois anos, e na atual $25,0 \%$ dos médicos e $28,5 \%$ dos enfermeiros estavam de um a três anos trabalhando no local.

Com relação à orientação para iniciar o trabaIho, $71,4 \%$ dos entrevistados dizem ter recebido, posto que, destes, $7,1 \%$ por meio de colegas de trabalho, apenas 3,5\% mencionam que receberam da coordenação e $32,1 \%$ relatam ter recebido orientações oriundas de cursos complementares, enquanto oito não descreveram qual a forma de orientação recebida.

Com relação às condições para execução do trabalho, abordando-se o espaço físico quanto à quantidade e qualidade do mesmo, $71,4 \%$ consideram o espaço adequado quanto à qualidade, porém as opiniões dividem-se quanto à quantidade, quando $53,5 \%$ referem que os espaços físicos no interior das UBSF são insuficientes.

Após a caracterização dos entrevistados aborda-se as temáticas em questões abertas, analisadas segundo os temas que emergiram.

Quanto às dificuldades no processo de trabaIho, os entrevistados apresentaram diversos aspectos, como o processo de trabalho interno da equipe, a 
relação com a equipe Nasf e outros fatores externos, destacando-se, em suas respostas, primeiramente a categoria "Sobrecarga de Trabalho", na qual consta a redução de recursos humanos e as atividades assistenciais em conjunto com as gerenciais, queixas mencionadas por enfermeiros, os quais têm a atribuição de gerenciar as unidades. Na mesma categoria aparece o excesso de demanda, especificado por consultas denominadas extras ou eventuais, que consistem em respostas mencionadas pela categoria profissional médico, a qual está mais diretamente ligada ao atendimento das demandas de consultas extras.

Também há destaque para "Serviços de Retaguarda", que aparecem em relação à indisponibilidade de vagas e à falta de contrarreferência relativa aos pacientes encaminhados e às "Características do Território", desde distância com a área central do município, os recursos sociais disponíveis, assim como o fato de a população atendida ser extensa.

A categoria "Gestão Municipal" emerge do relato dos profissionais de falta de apoio institucional, destacando-se a menção específica à Secretaria Municipal de Saúde.

A "Equipe" é trazida pelos entrevistados na falta de profissionalismo e de motivação. A equipe "Nasf" é também mencionada para apontar dificuldades no processo de trabalho, pelo curto tempo de permanência nas unidades, periodicidade de visitas dos profissionais e poucas vagas para atendimento compartilhado com a equipe básica.

As "Condições Materiais de Trabalho" para assistir à população também foram inseridas nesse contexto, ressaltando a falta de medicamentos e o espaço físico inapropriado, o qual também foi relatado em questão específica para tal.

A "Descaracterização da Estratégia de Saúde da Família", a qual pode se relacionar também com a pouca valorização, por parte de profissionais e da própria população, das ações de prevenção e promoção de saúde.

Apesar de pouco expressiva, a categoria "Usuário" tem sua importância pelo apontamento de pouca participação e adesão de pacientes às ações realizadas pela unidade de saúde. O último tema abordado - "Pesquisas" - refere-se à dificuldade em responder instrumento de coleta de dados de pesquisas científicas, o que foi referido por apenas um indivíduo. A análise de Dificuldades no Processo de Trabalho é apresentada no Quadro 1.
Quadro 1 - Dificuldades no Processo de Trabalho: categorias temáticas e exemplos de respostas

\begin{tabular}{|c|c|}
\hline Categoria Temática & Exemplos de Respostas \\
\hline $\begin{array}{l}\text { Sobrecarga de } \\
\text { Trabalho }\end{array}$ & $\begin{array}{l}\text { Organização } \\
\text { Conciliar atividades assistenciais } \\
\text { com gerenciais } \\
\text { Número reduzido de funcionários } \\
\text { Demanda } \\
\text { Demanda Espontânea }\end{array}$ \\
\hline $\begin{array}{l}\text { Serviços de } \\
\text { Retaguarda }\end{array}$ & $\begin{array}{l}\text { Falta de contrarreferência } \\
\text { Falta de referência para o } \\
\text { secundário (especialidades) }\end{array}$ \\
\hline $\begin{array}{l}\text { Características do } \\
\text { Território }\end{array}$ & $\begin{array}{l}\text { Distância da cidade } \\
\text { № excessivo de pessoas (grande } \\
\text { área de abrangência) } \\
\text { Recursos Sociais } \\
\end{array}$ \\
\hline Gestão Municipal & $\begin{array}{l}\text { Falta de apoio da gestão municipal } \\
\text { Falta de incentivo da SMS } \\
\end{array}$ \\
\hline Equipe & \begin{tabular}{|l} 
Falta de profissionalismo \\
Desmotivação dos profissionais
\end{tabular} \\
\hline Nasf & $\begin{array}{l}\text { Núcleo de apoio com alguns } \\
\text { profissionais de } 15 \text { em } 15 \text { dias } \\
\text { Poucas vagas para agenda } \\
\text { compartilhada do Nasf } \\
\text { Falta de profissionais integrados } \\
\text { à equipe (fisioterapia, psicologia, } \\
\text { assistente social, educador físico) } \\
\end{array}$ \\
\hline $\begin{array}{l}\text { Condições } \\
\text { Materiais de } \\
\text { Trabalho } \\
\end{array}$ & $\begin{array}{l}\text { Falta de recursos/medicamentos } \\
\text { Espaço físico limitado }\end{array}$ \\
\hline $\begin{array}{l}\text { Descaracterização } \\
\text { da ESF }\end{array}$ & $\begin{array}{l}\text { Descaracterização da Estratégia de } \\
\text { Saúde da Família } \\
\text { Desvalorização de ações de } \\
\text { prevenção e promoção à saúde }\end{array}$ \\
\hline Usuários & $\begin{array}{l}\text { Falta participação nas atividades da } \\
\text { unidade } \\
\text { Adesão às orientações prescritas }\end{array}$ \\
\hline Pesquisas & Responder questionário de pesquisa \\
\hline
\end{tabular}

Outra questão do instrumento aplicado refere-se às reuniões de equipe. Todos os respondentes consideram que elas são importantes para o desenvolvimento do trabalho. Durante o período do estudo essas reuniões eram feitas semanalmente. A maioria dos entrevistados $(85,7 \%)$ destaca que nestes espaços ocorrem discussões de caso, porém 14,3\% diz que em suas UBSFs são realizadas outras reuniões específicas para essa finalidade. Apenas um entrevistado diz que não há a participação de membros da equipe Nasf na reunião de equipe da UBSF.

Ao questionar as contribuições possíveis da atuação do fisioterapeuta para a saúde do usuário, foram obtidas respostas que demonstram conhecimento do trabalho desse profissional, expectativas 
positivas quanto ao cuidado prestado ao usuário e a contribuição para o trabalho da equipe, as quais puderam ser categorizadas como apresentado no Quadro 2.

A maioria das respostas refere-se à contribuição do fisioterapeuta na categoria "Recuperar a Saúde" do usuário, expresso em alusões a tratamento de modo geral ou específico, citando áreas da saúde, como Ortopedia, Neurologia ou Respiratória, destacando-se a referência à redução da dor. Em seguida, aparece outra categoria que aborda um dos objetivos do fisioterapeuta - "Promover Saúde" -, que abrange desde atividades de promoção à saúde até, também, prevenção de agravos.

A categoria "Melhorar a Qualidade de Vida" aparece como contribuição do fisioterapeuta a partir da sua intervenção, que também pode agir para "Co- laborar com a Equipe", outra categoria observada nas respostas. Essa temática traz aspectos como relacionamento com outros serviços, auxílio com a demanda, capacitações e discussões de casos, contribuindo para a qualificação do trabalho da equipe, presente na categoria "Melhorar a Qualidade da Assistência", que explicita também a diminuição do uso de medicações, dentre elas as analgésicas.

Quanto às expectativas relacionadas ao fisioterapeuta do Nasf (Quadro 3), as respostas destacam as "Atividades Coletivas", sendo estas voltadas a grupos específicos de tratamento ou de educação em saúde. Outro tema trazido pelos respondentes nesta questão é a "Qualidade da Assistência", referindo-se à resolutividade dos casos por meio de discussões e atendimento compartilhado, Também inserida nesse contexto está a motivação para o cuidado prestado. Ainda,

Quadro 2 - Contribuições do fisioterapeuta na saúde do usuário por categorias temáticas e exemplos de respostas

\begin{tabular}{|c|c|c|}
\hline Categorias Temáticas & Subcategoria & Exemplos de Respostas \\
\hline \multirow[t]{3}{*}{ Recuperar a Saúde } & Tratamento geral & $\begin{array}{l}\text { Melhora no quadro patológico } \\
\text { Tratamento de pacientes }\end{array}$ \\
\hline & Tratamento Especificado & $\begin{array}{l}\text { Melhora da capacidade respiratória } \\
\text { Reeducação postural } \\
\text { Reabilitação em casos de déficits neurológicos e motores } \\
\text { Tensão, estresse } \\
\text { Tratamento de tendinopatias, artroses, artrites, etc. }\end{array}$ \\
\hline & Redução da Dor & $\begin{array}{l}\text { Melhora de dores crônicas } \\
\text { Diminuição dos quadros álgicos }\end{array}$ \\
\hline \multirow[t]{2}{*}{ Promover Saúde } & Prevenção/Promoção & $\begin{array}{l}\text { Educação do paciente em relação a sua saúde } \\
\text { Atuar na prevenção } \\
\text { Atendimento com enfoque preventivo }\end{array}$ \\
\hline & Prevenção de Agravos & $\begin{array}{l}\text { Orientar atividades necessárias ou não no desenvolvimento dos } \\
\text { agravos } \\
\text { Orientar os já doentes } \\
\text { Prevenção de agravos }\end{array}$ \\
\hline $\begin{array}{l}\text { Melhorar a Qualidade de } \\
\text { Vida }\end{array}$ & \multicolumn{2}{|c|}{$\begin{array}{l}\text { Diminuição do sofrimento } \\
\text { Melhorar a qualidade de vida }\end{array}$} \\
\hline Colaborar com Equipe & \multicolumn{2}{|c|}{$\begin{array}{l}\text { Articulação com os outros equipamentos da rede } \\
\text { Diminuição das consultas eventuais dos "crônicos" } \\
\text { Atendimento multiprofissional compartilhado } \\
\text { Compartilhamento de saberes } \\
\text { Ajuda na demanda } \\
\text { Capacitação de equipe } \\
\text { Trazer um olhar mais amplo sobre a saúde } \\
\text { Discussão de casos }\end{array}$} \\
\hline \multirow[t]{2}{*}{$\begin{array}{l}\text { Melhorar a Qualidade da } \\
\text { Assistência }\end{array}$} & Cuidado & $\begin{array}{l}\text { Melhor qualidade de assistência } \\
\text { Melhorar o cuidado prestado ao usuário }\end{array}$ \\
\hline & Uso de Medicação & $\begin{array}{l}\text { Diminuir a prescrição de AINH (anti-inflamatório não hormonal) } \\
\text { Abordagem não medicamentosa }\end{array}$ \\
\hline
\end{tabular}

Fonte: Questionários de Pesquisa. 
são apontadas ações de matriciamento e compartilhamento de saberes, inseridas na categoria "Colaboração com a Equipe".

A "Reabilitação" é mencionada quanto à cronicidade das doenças e fisioterapia respiratória, assim como atividades de reabilitação em geral. Quanto à "Promoção/Prevenção", há a citação de ações de prevenção para usuários em idade escolar. Novamente a "Qualidade de Vida" é trazida, nesse contexto, como expectativa do trabalho do fisioterapeuta da equipe de apoio. A "Articulação com Outros Serviços" aparece, esperando-se que o profissional possa contribuir na ponte entre a atenção básica e os serviços especializados.

Por último, foi citada, de forma crítica, a periodicidade das visitas dos profissionais do Nasf na categoria "Carga Horária na Unidade".

Todos os participantes médicos relatam já ter realizado encaminhamento de pacientes para a fisioterapia, tanto ao setor secundário, quanto ao terciário, assim como conhecem o fluxo de encaminhamento e os serviços disponíveis no município. Parte dos participantes enfermeiros, $10,7 \%$, destacam não terem feito nenhum encaminhamento à fisioterapia, o que está condizente com o fato de que, muitas vezes, a atribuição de encaminhar pacientes aos serviços de retaguarda pode pertencer ao médico, dependendo da organização estabelecida pelo serviço de saúde.

Ao abordar a opinião que os profissionais entrevistados possuem dos serviços de retaguarda em fisioterapia do município, com relação ao número de serviços disponíveis, $50,0 \%$ dos médicos entrevistados apontam como ruim e 50,0\% dos enfermeiros como regular. Com relação ao número de vagas para atendimento, $57,1 \%$ dos médicos e $64,2 \%$ dos enfermeiros disseram que consideram inadequado. Já com relação à qualidade da assistência prestada, 50,0\% dos médicos e $57,1 \%$ dos enfermeiros apontam como boa.

Outro aspecto diz respeito às dificuldades existentes no encaminhamento realizado para os serviços de Fisioterapia: $82,1 \%$ dos participantes afirmam que os serviços de retaguarda não realizam contrarreferência para a APS, seguida da dificuldade de continuidade do tratamento $(64,3 \%)$, tema respondido principalmente pelos médicos, responsáveis diretos pelos encaminhamentos realizados.

Quadro 3 - Expectativas com relação ao fisioterapeuta do Nasf: categorias temáticas e exemplos de respostas

\begin{tabular}{|c|c|}
\hline Categorias Temáticas & Exemplos de Respostas \\
\hline Atividades Coletivas & $\begin{array}{l}\text { Atuações em grupos terapêuticos } \\
\text { Formação de grupo segundo a patologia } \\
\text { Grupos de lombalgia } \\
\text { Desenvolvimento de grupo para dor crônica }\end{array}$ \\
\hline Qualidade da Assistência & $\begin{array}{l}\text { Discussão de casos } \\
\text { Melhor qualidade de assistência } \\
\text { Resolutividade } \\
\text { Atendimento Compartilhado } \\
\text { Motivação }\end{array}$ \\
\hline Colaboração com a Equipe & $\begin{array}{l}\text { Matriciamento } \\
\text { Apoio matricial } \\
\text { Orientar o profissional sobre como proceder em alguns casos } \\
\text { Capacitação da equipe } \\
\text { Compartilhamento de saberes }\end{array}$ \\
\hline Reabilitação & $\begin{array}{l}\text { Resolução de casos crônicos } \\
\text { Desenvolver ações básicas de reabilitação } \\
\text { Fisioterapia respiratória } \\
\text { Atuação para pacientes acamados } \\
\end{array}$ \\
\hline $\begin{array}{l}\text { Promoção/ } \\
\text { Prevenção }\end{array}$ & $\begin{array}{l}\text { Promoção e prevenção à saúde } \\
\text { Orientações efetivas e reavaliação } \\
\text { Atuação junto ao escolar para prevenção }\end{array}$ \\
\hline Qualidade de Vida & Melhora da qualidade de vida \\
\hline Articulação com outros Serviços & $\begin{array}{l}\text { Referência para casos a serem encaminhados à Unesp } \\
\text { Avaliação para SADT para Fisioterapia }\end{array}$ \\
\hline Carga Horária na Unidade & Acho ruim o profissional vir a cada 15 dias \\
\hline
\end{tabular}


A pouca oferta de serviços disponíveis e a demora para conseguir atendimento são mencionadas por $42,8 \%$ dos entrevistados. Esses dados sugerem que há expectativas da equipe mínima quanto a mudanças para que o usuário seja bem-assistido.

Continuando na abordagem dos serviços de retaguarda do município, houve o questionamento referente à expectativa do profissional quando encaminha para o serviço de fisioterapia, conforme apresentado no Quadro 4.

A maioria das respostas refere-se à "Reabilitação", demonstrando que a visão da atuação fisioterápica, quando em setor secundário e terciário, é voltada para a recuperação física e funcional, e a redução do quadro álgico é também mencionada em grande escala, uma vez que apresenta grande demanda nas unidades de saúde. Em seguida, discute-se a categoria "Resolutividade", com relação à efetividade dos cuidados recebidos pelos usuários encaminhados. Nessa mesma categoria aparece o questionamento quanto à quantidade de sessões do tratamento fisioterápico, em razão da normatização de dez sessões, que podem ou não ser prorrogadas, e que não possibilita ao profissional que irá realizar o atendimento determinar a necessidade do tratamento de acordo com cada caso.
Novamente a "Contrarreferência" é mencionada, demonstrando o quanto essa é uma questão importante para a equipe da ESF, posto que são responsáveis pelo acompanhamento dos usuários da área de abrangência da unidade e, muitas vezes, não conseguem as informações necessárias ao bom cumprimento de suas funções. A "Melhora da Qualidade de Vida do Paciente" também é repetida nessa questão, assim como as ações de "Prevenção/Promoção". Neste momento visualiza-se que a atuação em promoção da saúde e prevenção de agravos pode ser realizada não somente na APS, mas também em serviços secundário e terciário. Por fim, a "Colaboração com a equipe" é outro aspecto mencionado.

Para construir um panorama das demandas em fisioterapia que mais surgem para os profissionais da ESF, foram elencadas algumas patologias para serem assinaladas, com a possibilidade de acrescentar outras, se necessário. Três categorias não foram citadas: "Doenças Cardíacas", "Doenças Vasculares" e "Malformações Congênitas". As principais citações referem-se aos quadros álgicos, como Lombalgia/Lombociatalgia, Cervicalgia/Cervicobraquialgia e Dorsalgia/Mialgia, como demanda mais recorrente, seguidas de Alterações Posturais que podem relacionar-se à Ortopedia,

Quadro 4 - Expectativa ao Encaminhar para Serviços de Retaguarda de Fisioterapia: categorias temáticas e exemplos de respostas

\begin{tabular}{|l|l|l|}
\hline \multicolumn{1}{|c|}{ Categorias Temáticas } & Subcategoria & \multicolumn{1}{|c|}{ Exemplo de Respostas } \\
\hline Reabilitação & Recuperação & $\begin{array}{l}\text { Sequelas do paciente } \\
\text { Reabilitação } \\
\text { Estabilização do quadro clínico } \\
\text { Redução das incapacidades }\end{array}$ \\
\cline { 2 - 3 } & Redução da Dor & $\begin{array}{l}\text { Redução dos quadros álgicos } \\
\text { Diminuição das dores crônicas } \\
\text { Melhora do quadro álgico }\end{array}$ \\
\hline Resolutividade & $\begin{array}{l}\text { Tratamento medicamentoso não é eficaz } \\
\text { Quem determina o tempo de tratamento é o fisioterapeuta } \\
\text { Problema seja resolvido a longo prazo } \\
\text { Resolução do quadro } \\
\text { Resolver a queixa do paciente } \\
\text { Demanda do paciente seja atendida } \\
\text { Avaliação prévia, um manejo competente }\end{array}$ \\
\hline Contrarreferência & $\begin{array}{l}\text { Contrarreferência } \\
\text { Devolutiva }\end{array}$ \\
\hline $\begin{array}{l}\text { Melhora da Qualidade de Vida do } \\
\text { Paciente }\end{array}$ & \begin{tabular}{l} 
Melhora da qualidade de vida do paciente \\
\hline Prevenção/ \\
Promoção
\end{tabular} & $\begin{array}{l}\text { Prevenir sequelas } \\
\text { Prevenção e promoção } \\
\text { Orientar o paciente para execução de suas atividades laborais }\end{array}$ \\
\hline Colaboração com a Equipe & $\begin{array}{l}\text { Diminuir consultas eventuais } \\
\text { Trabalho em conjunto }\end{array}$ \\
\hline
\end{tabular}

Fonte: Questionários de Pesquisa. 
Dores em Articulações sem Diagnóstico, Tendinopatia, Fibromialgia, Osteoartrose/Osteofitose, Esporão de Calcâneo, Doenças Reumatológicas e Epicondilite. Este resultado está concernente com as observações feitas pelos entrevistados referentes às contribuições do fisioterapeuta na saúde do usuário, quando citam a redução da dor, conforme apresentado anteriormente.

Somente após todas essas demandas relacionadas à dor surgem outros campos, como Doenças Respiratórias, Paralisia Cerebral, Incontinência Urinária, Desenvolvimento Neuropsicomotor, Deficiência Visual, Auditiva e/ou de Linguagem Falada e Doenças de Medula Espinhal. Por fim, aparecem Fratura e Gonalgia (dor no joelho), termo este utilizado, porém pouco conhecido, o que pode ter influenciado para ter sido pouco assinalado.

\section{DISCUSSÃO}

Os profissionais participantes da pesquisa revelaram possuir conhecimento dos recursos de fisioterapia e que os consideram de relevância no cuidado aos usuários dos serviços, daí o motivo de apontarem a necessidade de maior retaguarda nessa área. Tal reconhecimento parece estar relacionado à grande demanda referente a dores crônicas, conforme os entrevistados informaram. O reconhecimento de valor nos cuidados do fisioterapeuta está em consonância com o apontado na literatura, conforme encontrado em revisão integrativa realizada por Fonseca et al. (2016).

A fisioterapia alcança cada vez mais seu espaço em várias vertentes do cuidado ao usuário, e sua inserção na APS, apesar de recente, já permite um panorama de melhoria da assistência prestada pela ESF. Um estudo realizado por Oliveira et al. (2011) fez uma avaliação do conhecimento da equipe de saúde das unidades do município de Coari, no Estado do Amazonas, sobre a atuação do fisioterapeuta na APS. Os resultados mostraram que a maioria dos profissionais reconhece de forma mais assídua a atuação em serviços especializados sob a ótica da reabilitação, sendo pouco expressiva sua atuação em prevenção e promoção, apesar de o profissional ser generalista e estar apto para intervir nas diferentes vertentes do cuidado.

Para prestação do cuidado ao usuário, o cumprimento dos princípios e diretrizes preconizados do SUS é importante. O fisioterapeuta pode contribuir de forma clara para um sistema de saúde universal, equitativo, com a transformação do modelo de atenção, por meio das intervenções multiprofissionais, o cuidado integral e a construção e manutenção das redes de cuidado (FONSECA et al., 2016).

Os profissionais destacam, em muitos momentos, a contribuição do fisioterapeuta para a resolutividade dos casos e melhoria da qualidade de vida do paciente. As queixas crônicas, especialmente em relação aos quadros álgicos, consistem em grande demanda para as unidades, a qual sobrecarrega e causa impacto sobre as atividades realizadas, e esta situação necessita da colaboração do profissional de Fisioterapia (SANTOS et al., 2014). As ações de promoção e prevenção tiveram grande destaque nos relatos dos respondentes, percepção essa que contribui ainda mais para a interação entre a equipe da unidade de saúde e a equipe do Nasf.

A necessidade da consolidação das RAS tornou-se clara neste trabalho, pois os participantes lembram que, além de disponibilidade dos serviços de retaguarda, a comunicação entre cada área é de extrema relevância para o acompanhamento integral do usuário. A articulação com os serviços também foi referida como contribuição possível dos profissionais fisioterapeutas do Nasf. A periodicidade da presença da equipe do Nasf quinzenal é discutida e pode demonstrar a importância atribuída a esta equipe para o trabalho na UBSF.

A reorganização do processo de trabalho pode contribuir para ampliar a qualidade da assistência ofertada, a resolutividade e a situação de saúde da população. Também o custo-efetividade é um tema importante quando se trata de gestão em saúde, pois a APS necessita de apoio para o acompanhamento dos usuários, e o modelo "hospitalocêntrico" vigente por muito tempo contribuía de forma negativa para a expansão do SUS com qualidade, por exigir tecnologias mais custosas, além de demandar tecnologias desnecessárias na resolução de muitos casos (MENDES, 2011).

Apesar de pouco expressiva, a categoria "Usuário" tem sua importância pelo apontamento de pouca participação e adesão de pacientes às ações realizadas pela unidade de saúde. Essa visão dos profissionais de saúde, atribuindo ao usuário a responsabilidade pelas condições de sua saúde, também foi verificado no estudo de Silva e Sirena (2015). A "culpabilização" ao usuário não considera as reais razões que fazem com que o indivíduo não participe de ações promovidas pelas unidades, ou deixe de executar determinada orientação, sendo esta uma simplificação do cuidado (COELHO; SOARES, 2014). 
A recuperação da saúde ainda consiste na principal expectativa com relação ao trabalho realizado pelo fisioterapeuta. A fisioterapia advém de um período pós-Segunda Guerra, quando atuou nos mutilados e com déficit de funcionalidade por muitos anos, mantendo essa atribuição reabilitadora como único objeto de trabalho. Pode-se considerar que a fisioterapia é uma profissão nova, e que a reabilitação e prevenção de doenças ainda são as principais atribuições esperadas (BISPO JÚNIOR, 2010; MELO et al., 2018).

Como mencionado anteriormente, as consultas denominadas eventuais ou extras causam um impacto grande na demanda atendida, gerando, possivelmente, uma sobrecarga de trabalho e descaracterizando os principais objetivos da UBSF. A qualidade da atenção prestada ao usuário pode contribuir na diminuição dessa sobrecarga de trabalho, caracterizando as respostas mencionadas na categoria "Colaboração com a Equipe". Aliada a essa busca em qualidade dos serviços, a gestão municipal é fundamental para fomentar e impulsionar a ESF conforme seus princípios, incentivando os profissionais e usuários para a mudança do modelo de atenção, ampliando para ações que não sejam somente curativas (BISPO JÚNIOR, 2010; DIAS, 2013).

Os resultados demonstram que as queixas álgicas são recorrentes e que algumas possibilidades de intervenção do fisioterapeuta parecem ser menos conhecidas, como aquelas relacionadas ao desenvolvimento neuropsicomotor e sensorial.

As atividades coletivas são competências que devem fazer parte do rol de ações executadas pelo fisioterapeuta, pois consistem em formas de operar o trabalho, e possuem sucesso quando se trata de práticas corporais (BISPO JÚNIOR, 2010; BRASIL, 2010).

A qualidade de vida, apesar de ser um tema genérico, neste momento aparece como um objetivo a ser alcançado quando se trata das características funcionais do indivíduo, as quais devem ser mantidas ao máximo, e o fisioterapeuta possui, segundo os entrevistados, uma contribuição fundamental.

Vale ressaltar que a formação do profissional fisioterapeuta tem sofrido debates e incursões dentro do campo da saúde coletiva (FONSECA et al., 2016), e há grande contribuição quando se trata das mudanças nas Diretrizes Curriculares nacionais e as residências multiprofissionais (OLIVEIRA; MEDEIROS, 2018), com ampliação da formação de profissionais para atuação na APS.

\section{CONCLUSÃO}

Para os entrevistados, a fisioterapia possui a responsabilidade de resolutividade dos casos, concernente com as atribuições possíveis de cada serviço, mas também deve atuar com a promoção da saúde e prevenção de agravos. Os profissionais médicos e enfermeiros reconhecem essa assistência como importante para o cuidado integral da saúde dos usuários. Também consideram que, diante das demandas existentes nas Unidades de Saúde da Família, o fisioterapeuta da equipe Nasf contribui de forma significativa para a qualidade do cuidado. A equipe necessita de apoio diante das demandas, por exemplo, para as queixas álgicas citadas em vários momentos, mas também perante o perfil de adoecimento populacional na atualidade e das características do território de abrangência. Os serviços de retaguarda merecem atenção especial na discussão, pois devem relacionar-se com a APS para garantir a integralidade e a continuidade do acompanhamento ao paciente. Esses equipamentos possuem grande relevância para a assistência e para a resolutividade, especialmente quando se trata de queixas crônicas, que promovem idas repetidas do usuário ao serviço de APS, sobrecarregando a unidade e podendo criar na equipe um sentimento de frustração ao não conseguir resolver o sofrimento trazido pelo paciente.

Cabe ressaltar que o município possui unidades sem ESF que não foram estudadas, sendo este um fator limitante deste estudo.

Apesar dos limites de um estudo local como este, ele revela a importância da ação interdisciplinar para o alcance do cuidado integral e, nesse sentido, a importância do Nasf e, em particular, da inserção do fisioterapeuta na equipe, o qual contribui diretamente na atenção referente às dores crônicas, demanda cada vez mais recorrente nas unidades da APS em todo o país.

\section{REFERÊNCIAS}

AITH, F. et al. Os princípios da universalidade e integralidade do SUS sob a perspectiva da política de doenças raras e da incorporação tecnológica. Revista de Direito Sanitário, São Paulo, v. 15 n. 1, p. 10-39, mar./jun. 2014.

BARDIN, L. Análise de conteúdo. Lisboa: Edições 70, 1979.

BARROS, J. O. et al. Estratégia do apoio matricial: a experiência de duas equipes do Núcleo de Apoio à Saúde da Família (Nasf) da cidade de São Paulo, Brasil. Ciência \& Saúde Coletiva, on-line, Rio de janeiro, v. 20, n. 9, p. 2.847-2.856, set. 2015. 
BISPO JÚNIOR, J. P. Fisioterapia e saúde coletiva: desafios e novas responsabilidades profissionais. Ciência \& Saúde Coletiva, v. 15, sup 1, p. 1.627-1.636, jun. 2010.

BERTONCELLO, D.; PIVETTA, H. M. F. Diretrizes curriculares nacionais para a Graduação em fisioterapia: reflexões necessárias. Cadernos de Educação, Saúde e Fisioterapia, Porto Alegre, v. 2, n. 4, p. 71-84, nov. 2015.

BRASIL. Portaria no 2.979, de 12 de novembro de 2019. Institui o Programa Previne Brasil, que estabelece novo modelo de financiamento de custeio da Atenção Primária à Saúde no âmbito do Sistema Único de Saúde, por meio da alteração da Portaria de Consolidação no 6/GM/MS, de 28 de setembro de 2017. Diário Oficial da União, 13 nov. 2019. BRASIL. Ministério da Saúde. Política Nacional de Atenção Básica. Aprova a Política Nacional de Atenção Básica, estabelecendo a revisão de diretrizes para a organização da Atenção Básica, no âmbito do Sistema Único de Saúde (SUS). Diário Oficial da União, 21 set. 2017.

BRASIL. Ministério da Saúde (Brasil), Secretaria de Atenção à Saúde. Departamento de Atenção Básica. Diretrizes do Nasf: Núcleo de Apoio a Saúde da Família. Brasília: Ministério da Saúde, 2010.

CALICCHIO, M. G. M. S. et al. Cronicidade: experiência de adoecimento e cuidado sob a ótica das ciências sociais. $\mathrm{Ci}$ ência \& Saúde Coletiva, Rio de Janeiro, v. 23, n. 2, p. 669670, fev. 2018.

COELHO, H. V.; SOARES, C. B. Práticas na Atenção Básica voltadas para o consumo prejudicial de drogas. Rev. Esc. Enferm. USP, São Paulo, v. 48, n. spe, p. 111-119, ago. 2014.

COFFITO. Conselho Federal de Fisioterapia e Terapia Ocupacional (Brasil). Resolução 363/2009. São Paulo: Coffito, 2009.

CUBAS, M. R. et al. Avaliação da Atenção Primária à Saúde: validação de instrumento para análise de desempenho dos serviços. Saúde em Debate, Rio de Janeiro, v. 41, n. 113, p. 471-485, abr. 2017.

DIAS, M. D. A. Compreender o trabalho na Atenção Primária à Saúde para desenvolver ações em Saúde do Trabalhador: o caso de um município de médio porte. Rev. Bras. Saúde Ocup., São Paulo, 38 (127), p. 69-80, 2013.

FERNANDES DE AMORIM, J. et al. Percepção dos fisioterapeutas sobre sua atuação no núcleo de apoio à saúde da família. Revista InterScientia, João Pessoa, v. 5, n. 1, p. 105115, 4 dez. 2017.

FONSECA, J. M. A. et al. A fisioterapia na atenção primária à saúde: uma revisão integrativa. Revista Brasileira de Promoção à Saúde, Fortaleza, v. 29, n. 2, p. 288-294, abr./jun. 2016.

HEIDEMANN, I. T. S. B.; WOSNY, A. M.; BOEHS, A. E. Promoção da Saúde na Atenção Básica: estudo baseado no método de Paulo Freire. Ciência \& Saúde Coletiva, Rio de Janeiro, v. 19, n. 8, p. 3.553-3.559, ago. 2014.

IBGE. Instituto Brasileiro de Geografia e Estatística. 2010. Censo demográfico de 2010. Disponível em: https://censo2010.ibge.gov.br/. Acesso em: abr. 2014.
MELO, E. A. et al. Dez anos dos Núcleos de Apoio à Saúde da Família (Nasf): problematizando alguns desafios. Saúde em Debate, Rio de Janeiro, v. 42, número especial 1, p. 328340, set. 2018.

MENDES, E. V. As redes de atenção à saúde. 2. ed. Brasília: Organização Pan-Americana da Saúde, 2011.

MINAYO, M. C. S.; SANCHES, O. Quantitativo-qualitativo: oposição ou complementaridade? Cadernos de Saúde Pública, Rio de Janeiro, v. 9, n. 3, p. 239-62, jul. 2003.

MOROSINI, M. V. G. C.; FONSECA, A. F.; LIMA, L. D. de. Política Nacional de Atenção Básica 2017: retrocessos e riscos para o Sistema Único de Saúde. Saúde em Debate, on-line, Rio de Janeiro, v. 42, n. 116, p. 11-24, jan./mar. 2018.

OLIVEIRA, A. M. B.; MEDEIROS, N. T. Fisioterapia na residência multiprofissional em Saúde da Família: relato de Experiência. Sanare, Sobral, v. 17 n. 2, p. 91-99, jul./dez. 2018.

OLIVEIRA, G. et al. Conhecimento da equipe de saúde da família acerca da atuação do fisioterapeuta na atenção básica. Revista Brasileira de Promoção à Saúde, Fortaleza, v. 24, n. 4, p. 332-39, out./dez. 2011.

OMS. Organização Mundial da Saúde. The World Health Report 2008: primary health care, now more than ever. Geneva OMS, 2008. Disponível em: www.who.int/whr/2008/en/ index.html. Acesso em: abr. 2014.

RIBEIRO, C. D.; SOARES, M. C. F. Situações com potencialidade para atuação da fisioterapia na atenção básica no Sul do Brasil. Revista Panamericana de Salud Pública, Washington, v. 36, p. 2, p. 117-123, jul. 2014.

ROCHA JÚNIOR, P. R.; MOREIRA, L. M. Fisioterapia na estratégia de saúde da família: percepção dos usuários. Revista de Atenção à Saúde, São Caetano do Sul, v. 14, n. 50, p. 2125, out./dez. 2016.

RODRIGUES, M. J.; RAMIRES, J. C. L. Estratégia de saúde da família: desafios para sua consolidação em Uberlândia. Hygeia, Uberlândia, v. 11, n. 20, p. 153-166, jun. 2015.

SANTOS, M. L. M. et al. Competências e atribuições do fisioterapeuta na Atenção Primária à Saúde. Fisioterapia Brasil, São Paulo, v. 15, n. 1, p. 69-76, jan./fev. 2014.

SILVA, G. G.; SIRENA, S. A. Perfil de encaminhamentos a fisioterapia por um serviço de Atenção Primária à Saúde, 2012. Epidemiologia e Serviços de Saúde, Brasília, v. 24, n. 1, p. 123-133, mar. 2015.

VASCONCELOS, M. I. O. et al. Avaliação da resolutividade e efetividade da atenção primária à saúde: revisão integrativa de literatura. Sanare, Sobral, v. 17, n. 1, p. 65-73, jun. 2018. 